Proceedings of the 2010 Winter Simulation Conference

B. Johansson, S. Jain, J. Montoya-Torres, J. Hugan, and E. Yücesan, eds.

\title{
TOWARDS METHODOLOGICAL APPROACHES TO MEET THE CHALLENGES OF HUMAN, SOCIAL, CULTURAL, AND BEHAVIORAL (HSCB) MODELING
}

\author{
Andreas Tolk \\ Engineering Management \& Systems Engineering \\ Old Dominion University \\ Norfolk, VA 23529, USA \\ Wim Huiskamp \\ TNO Defence, Security and Safety \\ P.O. Box 96864 \\ 2509 JG, The Hague, THE NETHERLANDS \\ Harald Schaub \\ IABG mbh \\ Einsteinstr. 20 \\ 85521 Ottobrunn, GERMANY
}

\author{
Paul K. Davis \\ RAND Corporation \\ 1776 Main Street \\ Santa Monica, CA 90401-2138, USA
}

\author{
Gary L. Klein \\ The MITRE Corporation \\ 7515 Colshire Dr., M/S H505 \\ McLean, VA 22102, USA
}

James A. Wall

Texas Center for Applied Technology

3407 TAMUS, Texas A\&M University

College Station, TX 77843-3407, USA

\begin{abstract}
The international developments of the recent years resulted in a radical change of tasks the armed forces are conducting. Supporting M\&S methods and tools can no longer focus on attrition, movement, and warfighting operations, but need to address reconstruction, crisis prevention, police tasks, and related efforts that are conducted in collaboration with or in support of civil agencies and organizations. A "whole of society" approach is needed, focusing on human, social, cultural, and behavioral (HSCB) modeling. This paper summarizes the position papers of a group of international experts in this emerging domain looking a methodological support to define a body of knowledge, establish communities of interests, integrate operationally relevant data, and work towards a supporting framework, and was prepared in preparation of a panel discussion during the Winter Simulation Conference 2010.
\end{abstract}

\section{INTRODUCTION}

Human, social, cultural, and behavioral (HSCB) modeling is a fuzzy term that only recently has been introduced to cope with the challenges of supporting non-combat operations that include military organizations and that are characterized by reconstruction, crisis prevention, police tasks, and other related efforts that are conducted by military forces in collaboration with or in support of civil agencies and organizations. Related issues have been addressed in research and conferences on computer generated forces (CGF), human behavior representation (HBR), and behavior representation in modeling and simulation (BRIMS), but HSCB is trying to establish a more holistic approach that merges technical aspects as well as expertise from related and contributing disciplines, in particular human and social 
sciences. The community did not yet establish a generally accepted definition. Scientific communities normally establish disciplines dealing with such new challenges by defining the body of knowledge (BOK) governing questions and applicable solutions.

Such an effort did not yet take place in the HSCB domain and many terms are overloaded and even contradictive. It is accepted, however, that in order to address training and analysis issues of the new spectrum of military operations a much broader approach that includes human and social sciences to derive non-attrition based models is needed. However, to support this activity, a common approach is needed; a community of interest must be established that brings experts of all relevant domains together. Some first experiments in this direction are described in (Garrett, Tolk and Bacon 2009).

A term closely related to these efforts is "human terrain." Military forces are used to take terrain into consideration when planning and conducting their operations, as the environment significantly constraints the options of a decision maker. As traditional terrain influences movement and attrition, "human terrain" influences socio-psychological operations. Under the lead of the U.S. Army Training and Doctrine Command (TRADOC), human terrain ideas are developed to serve the joint community. The initial focus of human terrain is collecting and providing data about cultural and social aspects that potentially influence an operation and thereby enabling the decision maker to understand the highly complex local socio-cultural environment in which they are deployed and operating. Silverman (2007) already pointed out that such data needs to be used for new models as well. In operations in Iraq and Afghanistan, the U.S. Army is now deploying "Human Terrain Teams" that collect such data, but neither are such teams sufficiently modeled in current training systems to allow military decision makers to train their efficient use, nor are the data applied sufficiently well to support new simulation developments. A better understanding of concepts, doctrine, and data is needed, which must be part of the methodological approach on HSCB challenges searched for in the panel discussion supported by this paper.

Finally, it needs to be discussed if new models are needed, or if legacy models can be extended to close the capability gap identified by HSCB requirements. It is also possible to theorize about the use of a framework solution that would enable to compose new and existing services. How to build such a framework methodologically - using a bottom-up approach starting with technical agreements and interconnectivity standards, or using a top-down approach in which required necessary services and potential providers are derived from a conceptual model - is subject of ongoing research. As the domain of HSCB modeling is diverse in itself and uses models that are derived from very different hypotheses, in particular in the human and social domain, even a new M\&S philosophy of multi-models may emerge that allows real multi-stage and multi-phase approaches composing multi-scope, multi-resolution, and multi-structure models (Yilmaz et al. 2007) to contribute to a solution - or at least a better understanding.

This paper has been prepared in support of the subject matter expert panel discussion on methodological Approaches to meet the challenges of HSCB modeling. Five internationally recognized experts in the HSCB domain were invited. All invited experts were asked to use the following four questions as guidelines to structure their inputs and ideas on what is needed in general and what the modeling and simulation $(\mathrm{M} \& \mathrm{~S})$ can produce in particular to better approach solutions to the new "whole of society" approach.

- HSCB Knowledge: The academic community understands the BOK as a comprehensive and concise representation of concepts, terms, and activities that make up a professional domain. What needs to make it into a BOK for HCSB modeling?

- HSCB Community: The need for a Social Science and Irregular Warfare Communities of Interest (COI) has been formulated in several forums dealing with HSCB challenges. What are the main issues you see for such a COI and what are your recommendations to establish such a COI? Do we need new organizations to manage this, or can existing organizations support this endeavor?

- HSCB Data: The United States are using so called "Human Terrain Teams" to collect data in new operational areas. How these teams operate and what kind of data they collect is captured in 
handbooks. How can these data be used - if they are usable - to address challenges of HSCB modeling.

- HSCB Models: The NATO SAS- recommends a common IW framework that could be used to assess $\mathrm{M} \& \mathrm{~S}$ and establish requirements is needed to address HSCB integration issues. Will it be possible to enrich current M\&S solutions - such as JSAF, JCATS, and comparable national models used in the NATO training centers - with HSCB components and solutions; or are new approaches needed?

These questions were distributed as a guideline to support a structured and constructive discussion, but by no means were meant to limit the contributions of the invited experts. Furthermore, not all questions needed to be addressed.

The inputs collected from each expert have been compiled into the following five sections, leaving the contributions together to support better understanding of the expert approach reflected in the answers. The last section of this paper summarizes the main recommendations of the approaches.

\section{IMPROVING THE BOK TO INNFORM MODELING OF COMPLEX OPERATIONS - INCLUDING IRREGULAR WARFARE (P. K. DAVIS)}

Modeling population-centric activities requires serious use of social science. Fortunately, the social science literature has a great deal to offer. However, the literature is fragmented along boundaries between academic disciplines, between basic and applied research, and between qualitative and quantitative research. Progress is being made in pulling the strands together and in what follows I draw specifically on RAND work to offer suggestions about the body-of-knowledge challenge, as also specified in (Davis 2009) and (Davis and Cragin 2009).

Realistically, the research base is not mature enough to support a coherent expression of the body of knowledge. The uncertainties and disagreements are profound, on both subject-area facts and even the nature of evidence and the appropriateness of different methodologies. Those hoping to find a nicely compiled body of knowledge that can be used to write computer models will be disappointed. Further, they will often find that there are multiple competing "theories." And, even if a particular "theory" is chosen, it will be found upon inspection to involve numerous variants and uncertainties. For example, someone decides to model the political behavior of some social entity (e.g., an ethnic faction within a particular country) with expected-utility theory. That would be only one approach, one very different from an approach based on the assumption of leader-follower behavior or an effort to represent the more emotional behavior of groups following significant events. Even if the expected utility approach is taken, however, which such approach is intended? Is "utility" calculated by considering only economic benefits? Does it allow for valuing group interests, and for altruism? If the answer is yes, implying that all are considered, then how do the several factors combine, and on what basis? The issues, then, here are not just "data" in the sense that traditional modelers think of, but the myriad of rules and algorithms that constitute the models themselves.

For the subject of complex operations it is a serious error to approach social-science issues as though there is or will soon be an agreed phenomenological model along with an agreed data base to feed it. Further, it would be a serious error to approach modeling with the notion of reviewing a number of competing "theories" and then down-selecting so as to choose the "best" of the alternatives, after which programming and the search for data could be undertaken. One reason is that, as social scientists often are wont to say, "Look, everything is context dependent; you can't have a general model." That is probably not true, but the general model, if one can be constructed, will be complex as discussed below.

There are some significant shortcomings of a "data" approach. If we had control of numerous parallel universes, we could run social-science experiments, collect data and infer empirical, data-driven "models" that could be used predictively (albeit with likely stochastic features). We don't and we won't (simulation experiments with agent-based models will become very important over time, but they are certainly not "empirical information" in the sense of observation of reality). They will improve over time, but the complexities of social behavior and the current state of the related modeling art suggest that 
progress will take many years. We do possess a good deal of historical data to draw upon for insights about, e.g., terrorism or insurgency, but the data usually refers to events in many different countries, times, and types of context, which makes valid inference a very dubious proposition. Only in a few instances do we have historical data for what can be thought of as natural experiments (e.g., events over time in the same or very similar contexts). Even if we had a good deal more for a region of interest (as might be possible with a very, very long insurgency and a dedicated effort to collect data sensibly), datadriven modeling has severe shortcomings with respect to reasoning. To mention an example familiar to military analysts with long memories, such data-driven modeling correctly predicted for many years that airpower's effects on campaigns would be relatively small in terms of direct kills (e.g., 0.25 kills per sortie). The model was correct-until it wasn't! In a small portion of the 1991 Gulf war, air forces demonstrated awesome killing power against concentrated mechanized forces on roads. That was an element of the military transformation that has already taken place. We would be wise to seek empirical data aggressively and consistently, but we should be very wary about using results of minimal-theory data-driven models for reasoning about strategy or for explaining observations. Theory and causal models are needed and, as in much of the history of science, the best use of data will often be to help infer and test models that can then be applied to a far greater set of cases than those on which we have empirical data. Using such models with exploratory analysis across uncertainty can greatly inform decisions even when data is sparse.

As recommended in (Davis 2009), we should consider starting with humbleness. For those of us concerned with causal models, which are extremely desirable for reasoning about strategy, one way to proceed is to go back to basics, characterizing the considerable knowledge that we have in informative but minimally pretentious terms. One method my colleagues and I have been involves what we call factor trees. Such diagrams can be focused upon by an entire group of people easily, and then debated and iterated in real time. It can quickly facilitate communication and substantive discussion that tends to be convergent rather than divergent. This virtue of simple diagrams will be well familiar to modelers and builders of software, especially those who appreciate the value of design.

More noteworthy is that the factor trees take a significant step toward reconciling and integrating competing "theories," i.e., moving toward a more general theory (at least in the sense of a conceptual framework). Considering the left-most branch of the tree dealing with motivations for support of terrorism, we see a number of potential motivations. These include religious fervor, economic gain, coercion and intimidation, the desire to be part of an exciting activity or group, or a sense of necessity such as defense against an occupier. Each is supported by different strands of the literature. In a discussion session showing these simultaneously, it is natural for participants to accept (perhaps grudgingly because they prefer a single-factor "theory") that any of the factors might dominate in some circumstances. Examples come to mind and people may quickly agree that, yes, Hamas' motivations for terrorism are different from those of al Qaeda. And, yes, even within the al Qaeda network there are those who are religiously driven and those who are not. "Truth" is not in deciding which single-factor theory is right, but in recognizing the multiplicity of pathways to the same result. This gives analytic meaning to "it all depends on context."

Another feature of the factor-tree methodology is that, where possible, it attaches "ands" and "ors" to distinguish among factors that are perhaps more or less necessary for the effect of interest and factors that may reinforce each other, substitute for each other, etc. This aspect of the approach is at best an approximation (exceptions can almost always be found for what seems to be an "and" connection), but approximations can be very useful. In particular, if some factors are at least potentially connected by "ands," it means that influencing any one of them may (i.e., with luck) undercut the effect. For example, one might be able to reduce terrorism by reducing motivations, the sense of legitimacy, the availability of mechanisms, or the sense of acceptability of costs. Conversely, seeing factors connected by "ors" reminds us forcefully to expect adaptations and substitutions. Our original factor-tree work emphasized a static view of the factors operating at a snapshot in time. It is also essential, however, to represent dynamics (as in ongoing work related to stabilization). Factor trees are akin to simplified, modular, hierarchical 


\section{Tolk, Davis, Klein, Huiskamp, Schaub and Wall}

influence diagrams used in representing system dynamics. Again, a good deal is known from social science about such dynamics (e.g., establishing security is a sine qua non for progress on economics) but often in only very approximate, qualitative ways. Our approach, then, has been to highlight the most important features in simple diagrams rather than to leap immediately to a full, detailed specification. First approaches are published in (Davis 2006), although the work has been enriched since this paper. Thus, we have used simple causal loops to show particular issues. And, in more ambitious work, we have used multi-resolution influence diagrams to characterize more complex matters, still in "bite chunks" that can be understood by a wide range of participants in discussion. We have also parameterized the inputs to allow such models to "run" and to be predictive, but only in the sense of exploratory analysis to understand the implications of the conceptual framework and to better understand the possibility space.

The U.S. Joint Staff has pursued a somewhat similar approach, developing a causal framework and MIT-style System Dynamics model to illustrate U.S. Counterinsurgency doctrine. A subsequent study expanded the framework and model to represent key dynamics in Afghanistan. The work has been done in the Joint Staff's Warfighting Analysis Division, with the doctrine work initiated by Colonel (ret.) Walter Barge and conducted by Captain Brett Pierson with PA Consulting. The Afghanistan study was conducted by Lieutenant Colonel Samuel Mowery with PA Consulting and briefed in theater by CAPT Pierson with more recent briefings by Commander Djamal Pullom.

My own view on the way ahead in providing an increasingly solid body of knowledge to be used in the modeling and simulation communities is, as discussed above, to start with great humility and simplicity, working deliberately to first identify what is actually known, representing that as simply as possible so as to maximize comprehensibility and communication. More detail can be added on a module-by-module basis over time, while preserving the ability to work at the higher levels of abstraction rather than succumbing to the allure of elaborate bottom-up models dependent on dubious lower-level algorithms and a host of unavailable and in some cases unobtainable "data." Increased ambitiousness could be manifested in baby steps. such as

- learning more about how to characterize when (i.e., the type of context) in which different factors have relatively more or less effect;

- working out "combining rules" at the nodes of factor trees, on a module-by-module basis with appropriate involvement of social scientists (rather than, say, specifying the algorithm and asking them for the constants of the equations, such as weighting factors);

- representing the node-by-node algorithms in simple terms such as logic tables or other forms of pseudo code;

- moving toward multi-resolution influence diagrams that maintain the apparent simplicity and comprehensibility while in fact incorporating more of the complexity, but while continuing to suppress less important interconnections and feedbacks (i.e., using approximations).

As it happens, my colleagues and I have considerable experience in using related methods over the years, although originally for developing alternative models of adversary reasoning so as better to inform strategy, see, e.g., (Kulick and Davis 2003). Finally, the image of where we are going should be of supporting exploratory analysis under uncertainty and disagreement, and supporting development of strategies that are flexible, adaptive, and robust-i.e., well hedged and designed to facilitate wise adaptation. This may seem a platitude until one realizes the dominance of the paradigm of merely considering uncertainties, making what seem to be the best judgments, but then plunging ahead without much hedging. It is quite possible to do much better. The recommendation here actually would have profound implications. 


\section{LESSONS LEARNED IN THE CONTEXT OF NATO (W. HUISKAMP)}

In practice, the BOK is the set of knowledge and skills that is deemed necessary and relevant for a person working in a specific domain. In established fields, the basic knowledge is typically acquired at a university and must then be maintained by the faculty. In the case of an emerging field like HSCB, we typically find that people from different backgrounds find each other and apply their multi-disciplinary background and experience to some new field emerging from common problems and interests. The challenge is to build on this baseline of individual efforts and develop new knowledge, understandings and experience. In the domain of HSCB modeling, we currently find experts from the social sciences and from information technology. This includes in particular 'soft' sciences like sociology, psychology and anthropology as well as 'technical' sciences like information and software architectures. In addition, the expertise of military subject matter experts (SME) is required that bring in knowledge about command and control (C2) and Course of Action planning processes. One of the first activities of interest to HSCB modelers that should be tackled to develop the BOK is to agree on definitions of the relevant players, their roles/responsibilities and their range of possible interactions (values and range of responses to stimuli or events). The results of this effort should furthermore be represented in a conceptual model (graphical, details in interaction diagrams, state machines with transitions, etc). This common conceptual model will facilitate communication between experts and model and simulation developers. Conferences, journals, books, etc., are good means to develop and maintain the BOK and furthermore support creating the desired and necessary COI.

The challenge with HSCB research (and most other areas of research) is to bring existing domains together rather than create a new domain from scratch. New organizations tend to build walls, compete for funding with existing activities and reinvent the wheel. It may take decades before existing knowledge from one domain filters through into a so-called new domain. The key to successful cooperation between domains is: define common objectives and acknowledge the specific expertise of all partners. In the case of NATO RTO, the topic of HSCB should be jointly tackled by experts from the HFM, SAS and NMSG panels, rather than result in separate study groups, symposia etc., as recommended in (Tolk 2009). The way ahead is to investigate the challenges, define common objectives and address these collectively and iteratively. First panel overarching activities have been successfully launched.

We need to recognize and understand the socio-cultural cues from the operational environment and use them in the decision making process. The conceptual model provides guidelines and identifies the attributes that represent the state of the HSBC system we are trying to understand. The data used to gain these insights should be based on empirical observations, not only on hypotheses. The collected 'human terrain' data should provide these attributes. In particular in collaborative environments as NATO the sharing of information is pivotal for the common success.

New modeling efforts are necessary to address HSCB challenges. The models mentioned in the questions are mainly kinetic in nature and deal with force deployment, self-defense, maneuvering, etc. The timescale of these models and the MOEs used to assess the results are different from the typical HSCB issues that we need to consider, as many socio-psychological effects take weeks, months, or even years before they become evident. For the near term it seems most appropriate to use separate yet interoperable models and visualization tools. Initially, much work will be needed to investigate issues like interoperability and correlation between the different models. Considering the fact that the HSCB models are less well understood they will also need more 'adjustments' based on experiences in the field. This will require analysts 'in theatre' to assess and update the models when they are to be used for decision support or training. It seems unlikely given the complexity of the HSBC problems that we can run predictive models. The value is in trying to develop a better understanding of what is going on (sense making) and what the possible range of (unexpected) effects is as a result of own actions. 


\section{THE ROLE OF EXPERTISE IN HSCB MODELS (G. KLEIN)}

The HSCB BOK must merge a wide range of expertise inputs. Figure 1 illustrates this wide range of expertise that needs to part of the body of knowledge to put HSCB models into operational usage. The process of using these models is one of human translation or transformation of information, from the realworld into the models, and out of the models into real-world implications. Not illustrated is the initial step of selecting the right models to help assess the situation at hand. Ultimately, this requires a synthesis of expert knowledge about the models, operations knowledge about the information requirements we are trying to answer, and subject matter expertise about the area of operations.

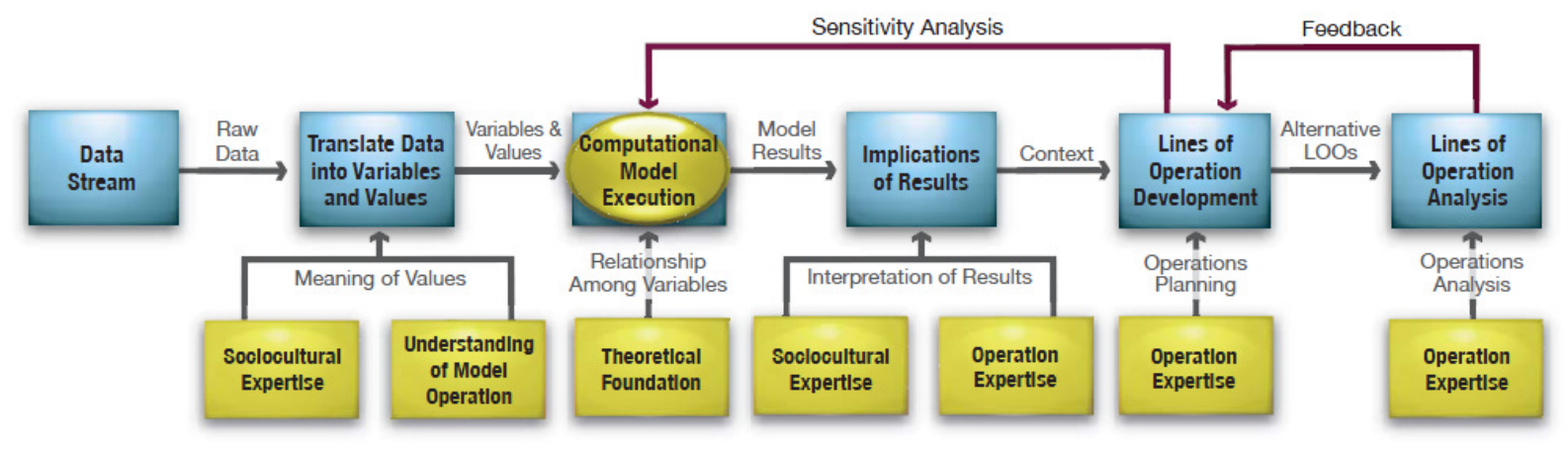

Figure 1: The Role of Expertise in HSCB Model Usage

For translating real-world data into the HSCB model world, almost invariably one must rely on people to bring their world knowledge and specialized expertise to bear.

For developing HSCB models, we rely on the expertise and methodologies of the various social sciences to assure us that these theories are the most descriptive and parsimonious explanations of available socio-cultural data. Subsequently, when translating these theories into computational models, social science expertise must be mixed with software engineering expertise to ensure a high quality translation - where the executable code is in fact an accurate expression of the theory.

For course of action planning under deep uncertainty one can shift from seeking optimality to seeking robustness. Robust planning uses exploratory modeling to forecast a landscape of plausible outcomes and then evaluates each outcome on its utility. This movement from optimal decision making to robust decision making is most significant. It is this shift in perspective that enables the most effective use of HSCB models. Consequently, expertise in this exploratory modeling use of HSCB models is crucial.

Determining the implications of the model results for the real-world situation needs modeling expertise (to understand the difference between changes in one agent versus another), psychological expertise to translate from the model to human constructs, and operations expertise to translate from human constructs into possible courses of action. Analyzing the feasibility of these courses of action requires additional operations expertise.

To support the usage of HSCB models in irregular warfare requires more than an informal community of interest. The integration of HSCB models into policy making, operational planning, or decision making will need to be a human collaborative process. It will need to choreograph a variety of expertise and organizations, which will need to be complemented by corresponding policies, procedures and information systems. A system of systems and an organization of organizations analogous to the Intelligence Community will need to be developed to operationally apply these models in an agile fashion and to have confidence in their contributions.

Information regarding local socio-cultural factors is needed at all stages of the military decision making process (MDMP) from initial analysis of the causes of threats or other adverse conditions, 
through the development and evaluation of alternate lines of operation (LOO), and the selection and implementation of a solution.

In addition, socio-cultural expertise is essential for base lining the status quo prior to intervention and then measuring effects after course of action implementation. The use of HSCB models as illustrated in Figure 1, does not change this need but can provide a structured way, through the models and their data structures, of recording and maintaining some of this information. These models can also help establish what information is truly discriminating for choosing among available LOO. Thus, continual interaction between socio-cultural assets, those developing the HSCB-model-based decision support systems, and the battle staff personnel using those systems is essential to guide the introduction of HSCB modeling into operational usage.

In contexts like IW, HSCB modeling information's primary purpose is to inform battle staff activities. Accordingly, the data that supports these systems needs to be as reliable as any other information on which the battle staff depends. Therefore, adequate data collection, integration, verification, and analysis by qualified personnel are as essential regarding social science derived information as any other inputs to the MDMP.

Two issues need to be addressed regarding the need for a unifying framework. First is whether there is a need for integrating HSCB models into a federation of models to support analysis. This type of tight integration involving reciprocal interdependence between models and the exchange of data during execution is only feasible in a small number of cases. However, my position it that decision support and analysis support can be addressed through lighter integration approaches that do not require a unifying interoperability framework. A pooled interdependence can be achieved by executing models separately and mapping their results to a common metric. When coupled with a robust decision making process, this approach can identify, from the multiple model perspectives, lines of operation or courses of action that will succeed across the broadest swath of plausible futures. Second is whether we need a comprehensive data model or ontology that will be common to all HSCB models and analysis. The breadth of social science domains that contribute to HSCB modeling, and the breadth of applications that these models can support already have their own terminology and frames of reference. These frames of reference serve the purposes of the practitioners in these areas. Therefore a comprehensive standard terminology or ontology that serves all of their needs does not seem to be practicable My position is that what is needed is a core ontology to which HSCB contributors and users can map their own terminology, frames of reference and data models. Such a core ontology can provide a Rosetta stone, by which translation among the different frames of reference can be achieved. Procedures and organizations will need to be established to maintain this core ontology.

In summary, the first issue that arises in each step of HSCB operational usage is the need for multidisciplinary expertise. The process of using these models is one of human translation or transformation of information, from the real-world into the models, and out of the models into real-world implications.

Consequently, conscientious operational usage will require development of policies, procedures and information systems to guide people in this endeavor and to ensure that their activities are audited and documented. In addition, training and doctrine will need to be developed to ensure decision makers are sensitive to the capabilities and limits of using such models of operational planning and policy development.

Of all the concepts presented in this section, the movement from optimal decision making to robust decision making is most significant. It is this shift in perspective that enables the most effective use of HSCB models. Ultimately, this usage of these models can enable planning even under circumstances of deep uncertainty. They will support the identification of more robust courses of action and policies, which will inherently improve the success of our decision making.

\section{ON THE NEED TO FOCUS ON THE HUMAN FACTORS (H. SCHAUB)}

Recent military documents of the Armed Forces increasingly focus on human being in mission planning, military operations and within the process of transformation. The impact of human factors in areas of 
military leadership, problem solving, threat assessment, and risk assessment is increasing especially in times of global threats and combined, network centric operations. Asymmetric threat confronts military personnel on all hierarchical levels with unfamiliar, yet unimaginable and unexpected environments and critical situations. There are no standardized solutions. Thus, the challenges for soft skills like thinking, planning and decision making are becoming more demanding.

Human scientists agree that mental and social processes take place in an environment characterized by complexity, uncertainty and continuous change. M\&S activities to reproduce mental-social-technical systems have to account for these characteristics. The application of $M \& S$ methods helps to make complexity, uncertainty and continuous change understandable and thus manageable. Integrating methods, theories and results from different scientific disciplines, IABG provides multiple interactive simulation systems of human behaviour, including mental and social processes and phenomena.

The scientific field of cognitive modelling represents mental processes of human informationprocessing (perception, thinking, planning, and decision making). The field of psychonics analyzes additionally human processes and phenomena of motivation, emotion, social interaction, and communication beyond cognitive parameters to understand the phenomena of human informationprocessing. The methods of serious gaming are used to efficiently develop realistic and interactive 3D scenarios. The combination of cognitive modelling, psychonics, and serious gaming results in simulation systems with virtual and interactive "agents". The implementation is carried out with the focal point on four areas, which are supported by Human-Factors. The important personality traits for current missions go far in excess of what has been captured by the scope of the diagnostic investigation. Human-FactorsAssessment Tools measure and evaluate necessary skills, like the acquaintance with complexity and incertitude.

It is necessary to use simulations as operations training instruments, to enhance effectiveness and efficiency of military schooling and training. The aim is to train situations which go beyond traditional exercises because of their complexity and their novelty over the prior art (e.g. specific assault scenarios of an asymmetric threat) or which are more cost efficient mapped by using a simulation tool (e.g. 100 simulated hooligans in a crowd and riot control (CRC) training instead of 100 volunteers).

To become optimal prepared for an unknown, undetermined place of action, it is important to know, how the place looks like, which persons are there, how they interact and how they e.g. react to sanctions of the action force. In order to achieve this it is important to get this knowledge not only in an abstract way, but also to have moved oneself in the city or territory with their typical inhabitants and tested specific behaviours through preparation. Therefore it is useful to implement interactive 3D scenarios of concrete locations, which are animated with simulated realistic acting agents.

Analysis and debriefing can be effectively supported through simulation of the behaviour of involved parties. It is necessary to design, implement, and use interactive 3D scenarios of anticipated and bygone crisis situations, to enable the action forces to analyse the behaviour of the involved peoples and parties under different conditions in a realistic simulated environment.

Modern theories and simulation concepts enable us to let the demand for the human in the focus of model building and simulations not become an empty phrase, but to support the armed forces with scientifically tested and user friendly tools in the area of requirements of protagonists from assortment over training and up to missions themselves.

\section{SELECTED COMPONENTS FOR AN HSCB ROADMAP (JAMES WALL)}

Regarding establishing a comprehensive and concise representation of concepts, terms, and activities it is important to gain an understanding as to how encompassing the body of knowledge related to HSCB modeling should be with respect to content. An HSCB BOK should support users ranging from novice to expert and from practitioner to researcher. As with any modeling endeavor there are issues associated with the specifics of the application domain and the technical means to model the desired aspects of the chosen domain to an acceptable level of fidelity. In the case of HSCB modeling the domain may represent a wide range of knowledge from a number of fields in the social sciences. Models that represent this 
domain knowledge will require both qualitative and quantitative methods. Because one purpose of the BOK is to provide the foundation for a common understanding, the diversity of disciplines needed to adequately represent models of populations and cultures strengthens the need for a BOK.

One approach to building a common understanding is the development of an ontology to provide a formal representation of the concepts related to HSCB modeling and to characterize the relationships that exist among those concepts. This development process must include contributions from representatives of all relevant disciplines.

Initially, the main challenge in developing HSCB COI may relate to inclusiveness with respect to attracting and maintaining the full range of professionals from the social sciences and engineering disciplines necessary to advance HSCB modeling and simulation environments. The community needs to quickly establish and promote a common vocabulary and converge on a common set of goals. These communities that are about increased understanding of diverse cultures are themselves a diverse collection of cultures given the broad array of disciplines needed to move HSCB modeling forward. I think that it is important that existing organizations provide the management necessary to promote HSCB COI. Military operations represent a continuum of non-kinetic and kinetic engagements and the appropriate management structure providing oversight to $M \& S$ standards and methods for training and analysis the full range of military operations should not be compartmentalized.

If currently available data can be used to establish an empirical foundation for more methodological approaches needs to be discussed. The original goal of the "Human Terrain Teams" (HTT) was to acquire relevant, socio-cultural information and knowledge and incorporate it into the decision making process in order to increase operational effectiveness. At some point it was decided that the data collected by the teams would be useful in generating scenarios to drive simulations that would be based on key cultural model parameters as well as actual events, situations, and activities that occurred in the operational area during the data collection timeframe. In turn, the output of the simulations could be useful in assessing (or perhaps "tuning") the fidelity of current models like the Joint Non-Kinetic Effects Model given that the outcomes of real operations are known. A study focused on making this assessment is ongoing now and no results have been published at this time.

Given the idea for using HTT data to assess the quality of current socio-cultural models came after the data was collected, a number of misalignments exist in terms of having all model inputs being supported by real world data. It is important to understand the impact of this misalignment by conducting a thorough sensitivity analysis for those input variables not sufficiently supported by the HTT data. In the future, it would be more useful for determining the efficacy of current socio-cultural models if HTT team members were trained to describe regional factions in cultural model terms and value ranges.

To address the need for HSCB modeling support, simulations are being extended today to address HSCB issues in two ways -HSCB models are federated with existing simulations - as described in (Henry 2009) - and new modules are directly integrated into current simulations like OneSAF. NASA JPL's Joint Non-kinetic Effects Model (JNEM) is a supporting model that is integrated, or federated, within the Joint Land Component Constructive Training Capability (JLCCTC). As a federate, JNEM monitors and interacts with the entity-based ground combat and maneuver land-war models such as the Corps Battle Simulation (CBS), Joint Conflict and Tactical Simulation (JCATS), and Warfighter Simulation (WARSIM). JNEM funnels human-readable output to the training audience via the Independent Stimulation Module (ISM). JRAM implements the National Simulation Center's Regional Analysis Model (RAM) for cultural population dynamics and the TRADOC HUMINT methodology for population cooperation modeling. The integration of both JNEM into JLCCTC and the Hybrid IW/IED Network-Defeat Toolkit $\left(\mathrm{HI}^{2} \mathrm{NT}\right)$ model into One Semi-Automated Force (OneSAF) provide positive examples of enriching current M\&S training exercise solutions with HSCB components. Overall, this approach is not a complete solution as there are still issues with mismatches in data fidelity and time resolution.

Another approach is to depart from the real-time training exercise mindset and pursue the development of standalone analytical simulators that extend beyond the traditional combat operations 
focus of force-on-force models over short simulation timeframes. As real life military activities/decisions are coupled to political, economic and cultural interventions with impacts that take months or years to materialize, a new simulation paradigm may be in order. One such example is NASA JPL's development of Athena which integrates a civilian population model, political model, economic model and information model into a standalone analytical simulator application that models interactions and allows situations to evolve over months to years.

\section{SUMMARY}

The new domain of HSCB modeling has initially been driven by the accomplishments of individuals and is gaining more and more interest within the M\&S community. In order to be successful, the various approaches must be captured contributing to a better methodological approach.

On the technical side, it is highly unlikely to be able to address all problems with one common approach. It is much more likely that the multi-simulation approach based on multi-resolution, multistage, and multi-models envisioned by Yilmaz et al. (2007) needs to be exploited to support the analysis of these multi-facetted challenges we are faced with as a community. Furthermore, integration methods are needed allowing to utilize the models we already use successfully.

Methodologically even more demanding is the observation that HSCB is a conceptual challenge that needs to be addressed on the modeling level. Technical solutions will become valuable enablers, but they will not contribute to a better understanding. It is in particular possible that we need to "get back to the drawing board" to get a better understanding of the problem and possible solutions. Identifying the right members of a COI to contribute to the HSCB BOK remains the main issue. A common terminology to discuss different contributing worldviews, not to be confused with a common model to deal with all challenges, is the first step in this process. We need the diversity of approaches to cope with the diversity of analytical challenges.

The panel discussion during the conference of these proceedings will hopefully successfully contribute to setting a necessary research agenda.

\section{REFERENCES}

Davis, P. K. 2006. A Qualitative Multiresolution Model for Counterterrorism. Proc. SPIE, International Society for Optical Engineering, Vol. 6227 (paper 15)

Davis, P. K. 2009. Specifying the Content of Humble Social Science Models. Proc. Summer Computer Simulation Conference, July 13-16, Istanbul, Turkey, pp. 452-461

Davis, P. K., and K. Cragin (Eds.). 2009. Social Science for Counterterrorism: Putting the Pieces Together. MG-849. Santa Monica, CA: RAND

Garrett, R. B., A. Tolk, and T. J. Bacon. 2009. Exploring Effective Methods for Modeling a Comprehensive Approach to Political, Military, Economic, Social, Information, and Infrastructure (PMESII) / Human, Cultural, Social, Behavior (HSCB) Community of Interest (COI). Proc. Winter Simulation Conference, Dec 13-16, Austin, TX, pp. 2860-2866

Henry, H. 2009. A Non-Kinetic Effects Federate for Training Simulations, Journal of Defense Modeling and Simulation 6(3):112-119

Kulick J., and P. K. Davis. 2003. Modeling Adversaries and Related Cognitive Biases. RP-1084. Santa Monica, CA:RAND

Silverman, B. G. 2007. Human Terrain Data - What shall we do with it? Proc. Winter Simulation Conference, Washington, DC: Dec 9-12; pp. 260-265

Tolk, A. 2009. Emerging M\&S Challenges for Human, Social, Cultural, and Behavioral Modeling. Proc. Summer Computer Simulation Conference, July 13-16, Istanbul, Turkey, pp. 462-469

Yilmaz, L., T. Ören, A. Lim, and S. Bowen. 2007. Requirements and Design Principles for Multisimulation with Multiresolution, Multistage Multimodels. In Proceedings of the 2007 Winter 
Simulation Conference, eds. S. G. Henderson, B. Biller, M.-H Hsieh, J. Shortle, J. D. Tew, and R. R. Barton, 823-832. Piscataway, New Jersey: Institute of Electrical and Electronics Engineers, Inc.

\section{AUTHORS' BIOGRAPHIES}

ANDREAS TOLK is an Associate Professor of Engineering Management and Systems Engineering at Old Dominion University. He is also affiliated with the Virginia Modeling Analysis and Simulation Center. He holds a M.S. and Ph.D. in Computer Science from the University of the Federal Armed Forces in Munich, Germany. He has published over 150 journal and conference papers, with more than 30 of them receiving honors and awards. His research focuses on developing mathematical models supporting M\&S composability and system of systems engineering. Key areas of interest are deriving open standards from consistent mathematical models that are applicable to support intelligent systems as well as the integration of $M \& S$ applications into operational systems for decision support. His email address is $<$ atolk@odu.edu>.

PAUL K. DAVIS is a senior principal researcher at RAND and a professor of policy analysis in the Pardee RAND Graduate School. He received his bachelor's degree from the University of Michigan and a Ph.D. in chemical physics from the Massachusetts Institute of Technology. He has been at RAND for many years, before which he was a senior executive in the Office of the Secretary of Defense. His research areas include strategic planning, decision science, and advanced methods of analysis and modeling (including conceptual modeling of social-science for counterterrorism and stabilization campaigns). His email is <pdaviserand.org $>$.

WIM HUISKAMP is Chief Scientist Modelling, Simulation and Gaming in the M\&S department at TNO Defence, Security and Safety in the Netherlands. He received a M.Sc. degree in Electrical Engineering from Twente University of Technology, The Netherlands. His research areas include system architecture, distributed real-time simulation and C2-Simulation interoperability problems. Wim acted as project lead for several national and international simulation (interoperability) projects and he leads TNO's research programme on Live, Virtual and Constructive Simulation, which is carried out on behalf of the Dutch MOD. Wim is a member of the NATO Modelling and Simulation Group (NMSG) and acted as member and chairman in several NMSG Technical Working groups. He is currently Chairman of the NMSG M\&S Standards Subgroup (MS3) and is the liaison of the NMSG to the Simulation Interoperability Standards Organization (SISO). His email is <wim. huiskamp@tno.nl>.

GARY L. KLEIN received his BA in Psychology, and his $\mathrm{PhD}$ in cognitive social psychology. His work has focused on modeling how people acquire and use information. Currently, he leads a number of projects on using simulation models to improve decision makers' "option awareness" under deep uncertainty. He is part of MITRE's team providing systems engineering support to the Director, Defense Research and Engineering's HSCB program. He is the Senior Principal Scientist in cognitive science and artificial intelligence in the $\mathrm{C} 2$ Center at The MITRE Corporation. His email is <gklein@mitre.org $>$.

HARALD SCHAUB serves as the Head of Human Factors and Man-System-Integration Department for the IABG (Munich, Germany) and is Professor for Psychology and Methodology at Otto-Friedrich University (Germany). He holds a Ph.D. in Psychology from the University of Bamberg (1992). He works on the topics of Human Factors in Defense and Security, in particular on Job Analysis in Command and Control Systems, Human Factors/Human Failure Analysis, Cognitive Agents Modeling and Simulation, Operation Analysis, CD\&E, psychological NCO/JDO-Capability, Assessment and Training . His e-mail is $<$ schaubeiabg. de $>$. 
JIM WALL currently serves as the Director of Computing and Information Technology for the Texas Center for Applied Technology and is an Associate Research Professor in the Industrial and Systems Engineering Department at Texas A\&M University. He holds a M.S. in Systems Technology (Command, Control, and Communications) (1986) from the Naval Postgraduate School and a Ph.D. in Computer Science (1993) from Texas A\&M University. He has extensive experience in command and control systems, distributed simulation environments, and virtual reality applications. He retired from the U.S. Army after 22 years of service where his last assignment was as a Senior Computer Scientist with the Army Research Laboratory. He was recently designated as a Regents Fellow by The Texas A\&M University System Board of Regents. His e-mail address is $<j i m-w a l l e t a m u$. edu $>$. 